\title{
Erratum
}

\section{Thermal conductivity as a probe of unconventional superconducting gap}

\author{
H. Aubin ${ }^{1}$, K. Behnia ${ }^{1}$, M. Ribault ${ }^{1}$, L. Taillefer ${ }^{2}$, R. Gagnon ${ }^{2}$ \\ ${ }^{1}$ Laboratoire de Physique des Solides (associé au CNRS), Université Paris-Sud, F-91405 Orsay, France \\ 2 Physics Department, McGill University, Montréal, Québec,Canada H3A 2T8
}

Z. Phys. B 103, 148-151 (1997)

Unfortunately the figures of the paper were not printed correctly. In particular, Fig. 1 was not printed at all, leading to an incorrect arrangement of the following figures. Because of this error, we reprint the entire paper here.

\begin{abstract}
We have carried out a comparative study of the thermal conductivity of two rectangular single crystals: a niobium sample and a detwinned YBCO. Thermal conductivity was measured as a function of the relative orientation of the crystaline axes and a magnetic field applied parallel to the sample plane. Different angular variations, two-fold for $\mathrm{Nb}$ and four-fold for YBCO, were observed in the two case. We discuss the microscopic effects related to the gap structure which are at the origin of this qualitative difference in the behavior of thermal conductivity in these two superconductors.
\end{abstract}

PACS: 74.25.Fy; 74.72.Bk; 72.15.Eb

One of the main subjects of current research on various families of exotic superconductors is the identification of the symmetry of their superconducting order parameter. Indeed, the knowledge of the supplementary symmetry broken in addition to the usual broken gauge symmetry is a preliminary step toward the understanding of the origin of the superconductivity in these compounds. Among the different experimental probes used to investigate the superconducting gap in high-temperature superconductors, one can mention the magnetic penetration depth[1], angular resolved photoemission[2], nuclear spin relaxation rate[3] and most recently quantum phase interference experiments[4] which have provided strong evidence for a $\mathrm{d}_{x^{2}-y^{2}}$ order parameter[5].

Thermal conductivity has been a very useful source of information on the structure of the superconducting gap in another unconventional superconductor, the heavy-fermion compound $\mathrm{UPt}_{3}[6]$. In this case, however, the predominance of the electronic contribution to the thermal conductivity at the onset of the superconductivity and down to temperatures well below $T_{c}$ is a major factor in the relevance of this

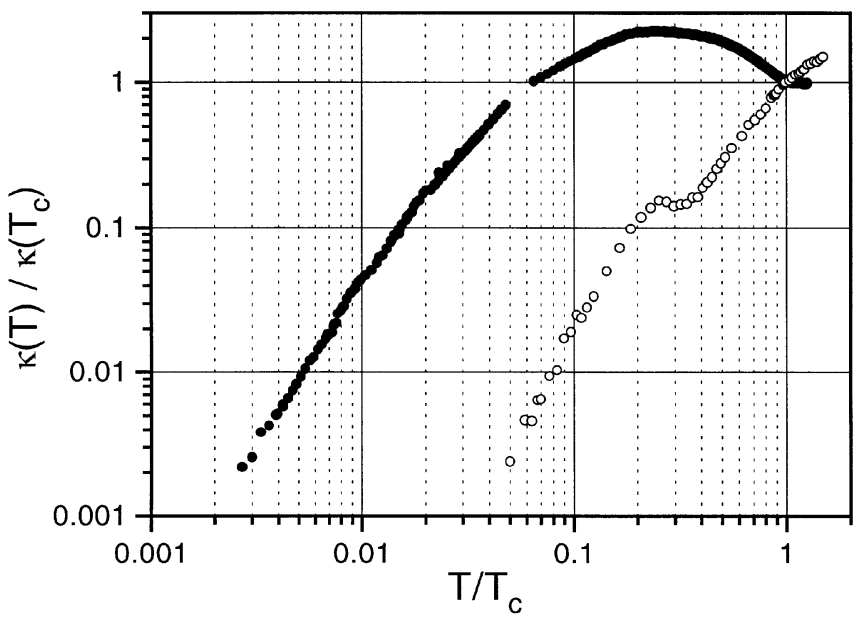

Fig. 1. Normalised thermal conductivity vs. normalized temperature for $\mathrm{YBCO}$ and $\mathrm{Nb}$

technique to explore the superconducting gap. The situation is very different in the high- $\mathrm{T}_{c}$ cuprates where at the onset of the superconductivity lattice vibrations have a substantial role in heat conduction. Nevertheless, as we will see below, thermal conductivity in high- $\mathrm{T}_{c}$ superconductors does contain important information on the k-space anisotropy of the superconducting gap.

In this short paper we report on a study of the thermal conductivity and its angular variation in optimally doped $\mathrm{YBa}_{2} \mathrm{Cu}_{3} \mathrm{O}_{7-\delta}(\mathrm{YBCO})$. To extract the unconventional features of superconductivity in this system, identical measurements were performed on a niobium crystal of the same shape and comparable size as a typical conventional superconductor.

Figure 1 shows the thermal conductivity of the YBCO and $\mathrm{Nb}$ samples as a function of normalized temperature. Thermal conductivity behaves differently in the two compounds. In the case of $\mathrm{Nb}$, thermal transport at the normal state is dominated by electronic heat carriers. The opening of the gap induces a drastic decrease in the density of electronic excitations, which is at the origin of the drop in the thermal conductivity at the superconducting transition. Another consequence of such a decrease is the reduction of the 

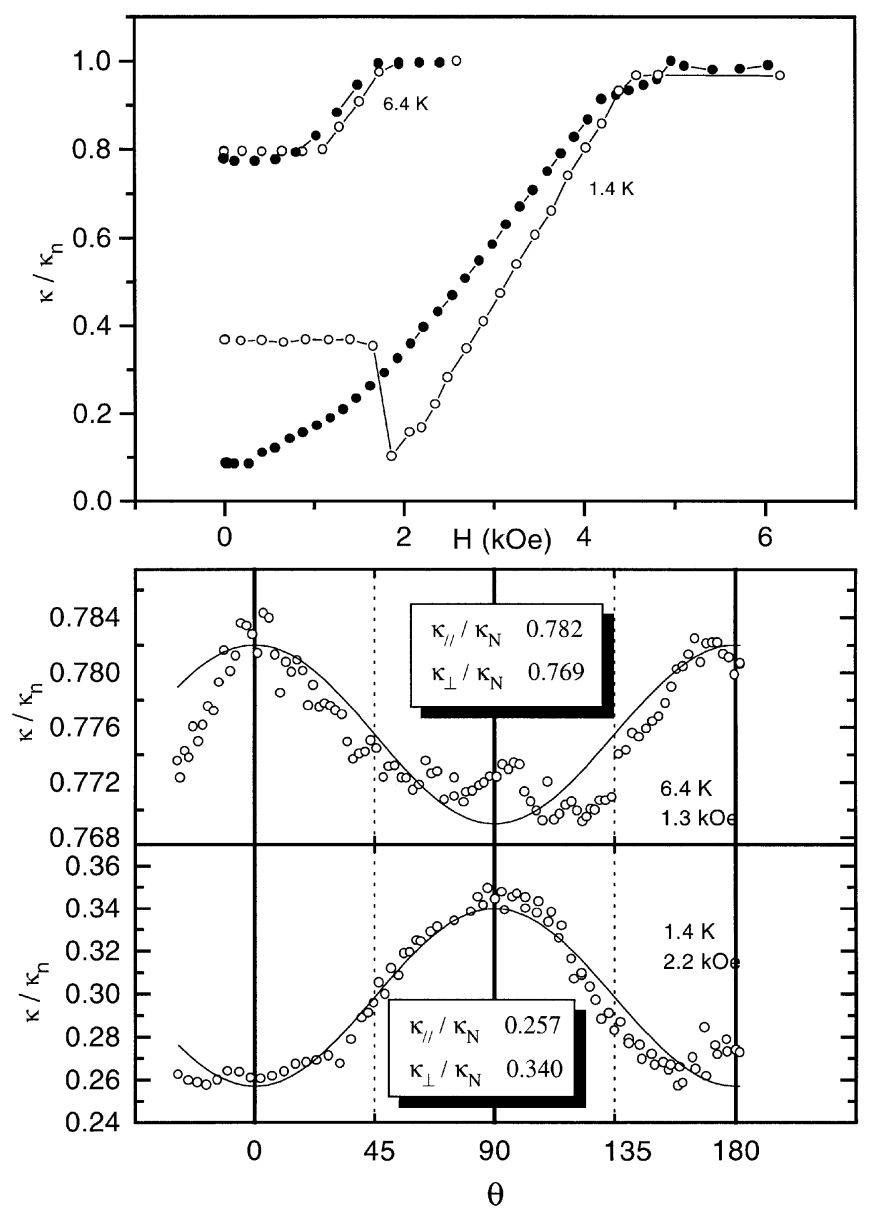

Fig. 2. a Field dependence of thermal conductivity for $\mathrm{Nb}$. b Angular variation of thermal conductivity for two different temperatures. Note the two-fold symmetry and the change in the sign of anisotropy

electronic scattering of phonons which leads to an increase in the lattice thermal conductivity and a peak in $\kappa$ at $\mathrm{T} / \mathrm{T}_{c}=$ 0.3 . At very low temperatures, the heat transport is completely dominated by the phonons. The situation is very different in the case of YBCO. Thermal conductivity increases suddenly at the superconducting transition passing through a maximum at $\mathrm{T} / \mathrm{T}_{c}=0.25$. At first, this was interpreted in a conventional scheme, where the effect of the superconducting transition on a lattice-dominated thermal conductivity would be an increase in the phonon thermal conductivity due to strong electron- phonon coupling[7]. Later, an alternative scenario was suggested[8] where, due to the strongly suppressed quasiparticle scattering rate in the superconducting state, the electronic contribution in the $\mathrm{Cu}-\mathrm{O}$ planes increases rapidly below $\mathrm{T}_{c}$. Early evidence for this scheme came from microwave conductivity data where a similar peak was observed. More recently, thermal Hall effect measurements[9] confirmed the increase in the electronic component of the thermal conductivity at $\mathrm{T}_{c}$ and nowadays a consensus slowly emerges on a partial electronic origin of this maximum in $\kappa$ [10]. The significant electronic contribution to thermal conductivity in the superconducting state is one reason to use this experimental probe to explore the angular distribution of the quasi-particle momentum and hence the structure of the superconducting gap.

Recently, Salamon and co-workers[11, 12] attempted to perform such an exploration by measuring the angular variation of the thermal conductivity $k(\theta)$ for a magnetic field rotating in the $\mathrm{Cu}-\mathrm{O}$ planes. They argued that thanks to the Andreev scattering of the quasi-particles by in-plane vortices, such a study can be a relevant probe of the k-anisotropy of the superconducting gap. Here we report on similar measurements leading to the first observation of a four-fold symmetry of $k(\theta)$, a qualitative signature of d-wave superconductivity. Moreover, by studying a Nb sample of comparable size and same shape we try to exclude any morphological origin for the effect as suggested by Klemm et al.[13] in their comment on [12].

We begin by looking at the effect of the magnetic field on the thermal conductivity of a conventional superconductor. Figure $2 \mathrm{a}$ shows the variation of the thermal conductivity $\kappa(H)$ for the $\mathrm{Nb}$ crystal, with the magnetic field applied perpendicular to the heat current. When the magnetic field is lower than the lower critical field, $\mathrm{H}_{c 1}$, no magnetic flux is present within the sample and the thermal conductivity remains insensitive to the field. Above $\mathrm{H}_{c 1}$, the magnetic field penetrates the sample and the BCS-like quasi-particles are scattered by the vortices, which produces a dip in $\kappa(H)$. Figure $2 \mathrm{~b}$ shows the angular variation of the thermal conductivity $\kappa(\theta)$, for the two low and high temperature limits. In the high temperature limit (close to $\mathrm{T}_{c}$ ), thermal conductivity is minimal (maximal) for the field applied perpendicular (parallel) to the heat current. This anisotropy is inverted in the low temperature limit. This angular variation of thermal conductivity and its temperature dependence was predicted by Maki[14], and confirmed experimentally by Lowell and Sousa[15].

Basically, the effects of the magnetic field on the quasiparticle excitation spectrum is understood through the Doppler shift of the electronic energies, $\mathrm{E}=\mathrm{E}_{0}-\mathbf{p} . \mathbf{v}_{s}$, due to the existence of a non-zero superfluid velocity $\left(\mathbf{v}_{s}\right)$ around the vortices. This superfluid flow originate two main physical process. The first is a pair-breaking effect, which occurs in the neighborhood of the vortices, particularly important for the electrons travelling parallel to the superfluid flow, i.e. perpendicular to the vortices. Therefore, at low temperature, when these gapless excitations dominate the gapped BCSlike excitations, the thermal conductivity is minimal (maximal) for the magnetic field perpendicular (parallel) to the heat current.

However, at high temperature, the dominant effect of the vortices is to scatter the common BCS-like excitations. Indeed, when a quasiparticle approaches the vortex core, its energy in the superfluid frame decreases down to the energy gap and then it is converted to a quasi-hole, halting its contribution to the heat transport. In their analysis of the coreless vortices lying between the $\mathrm{Cu}-\mathrm{O}$ planes of the high temperature superconductors, $\mathrm{Yu}$ et al.[12] rephrased this scattering mechanism in terms of Andreev reflection[16] which is a processus of retroreflection of excitations due to spatial variations of the amplitude or the phase of the order parameter. A peculiar feature of this scattering process is its strong dependence on the relative orientation of the quasi-particle momentum and the superfluid velocity, which is imposed by the magnetic field. Indeed, no Andreev reflec- 

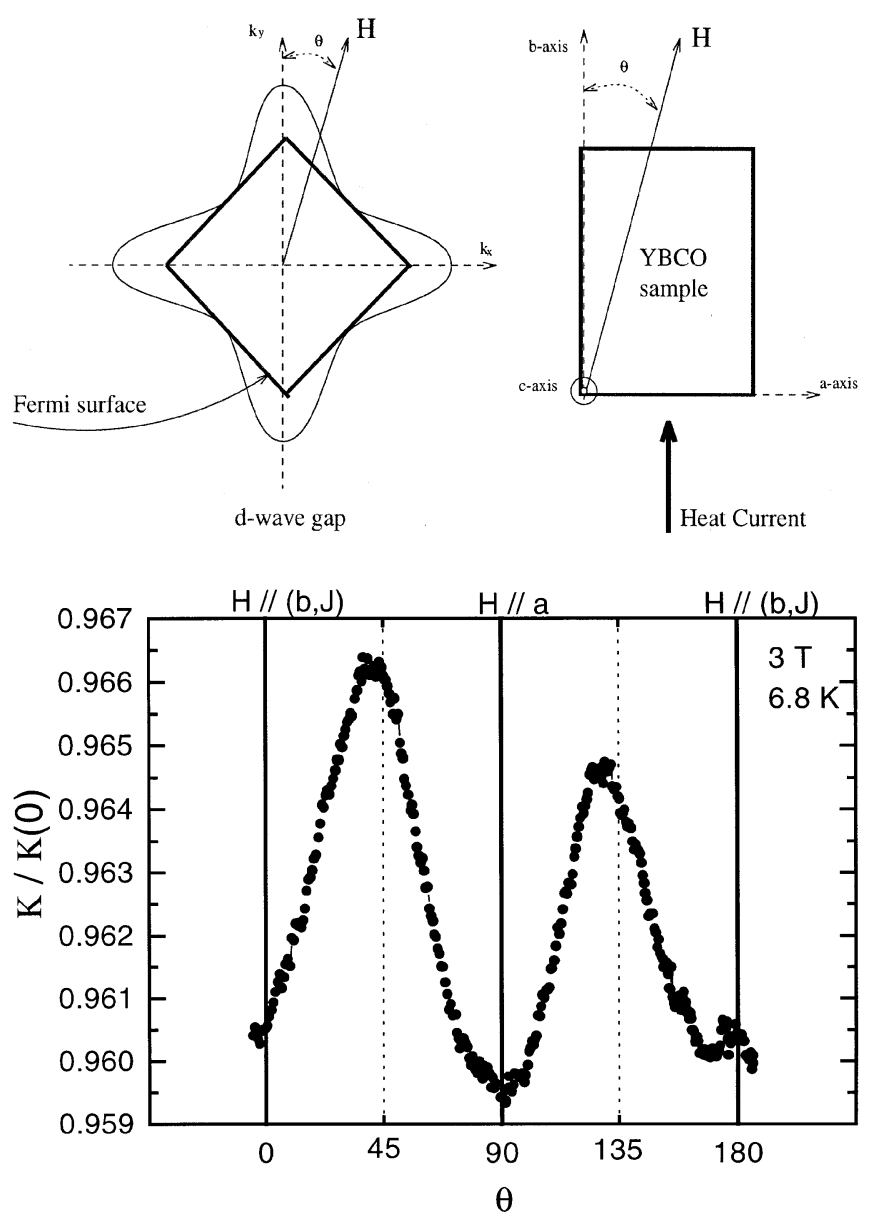

Fig. 3. a The anisotropic gap associated with a $\mathrm{d}_{x^{2}-y^{2}}$ superconducting order parameter has nodes at $k_{x}=k_{y}$. The magnetic field rotates in the a-b plane while the heat is injected along the b-axis. b The angular variation of thermal conductivity shows a clear four-fold symmetry. Maxima are observed when the field is along a nodal direction

tion occurs when the quasi-particle momentum is normal to the supercurrent and parallel to the magnetic field.

Therefore, due to the directionality of this scattering mechanism, thermal conductivity can identify the preferential momentum orientations of the excitations and the angular position of nodes or minima in the superconducting gap. In the case of the $\mathrm{Nb}$ crystal, due to the constant s-wave gap, the angular distribution of excitations above the superconducting gap is imposed by the direction of the applied heat current. Consequently, in the high temperature regime, the thermal conductivity is minimal (maximal) when the magnetic field is perpendicular (parallel) to the heat current. This is why, as seen in Fig. 2, the thermal conductivity in $\mathrm{Nb}$ in the two regimes has a twofold symmetry governed by the relative orientation of the applied heat current and the magnetic field.
Figure 3 shows that this angular variation is qualitatively different in the case of the YBCO crystal, where the angular distribution of the quasi-particle momentum does not follow the applied macroscopic heat flow and is imposed by the $\mathrm{k}$-space structure of the gap with a maximal density of excitations in the directions of nodes. We resolve a fourfold variation with maxima for odd multiples of $45^{\circ}$. This indicates that there are maxima in the angular distribution of the quasiparticle excitations in the vicinity of $\mathrm{k}_{x}= \pm \mathrm{k}_{y}$ so that when the field is aligned along these particular directions, most quasi-particles are not Andreev reflected, and the thermal resistance is lower. This is consistent with a gap structure associated with the so-called $\mathrm{d}_{x^{2}-y^{2}}$ superconducting order parameter. The thermal dependence of the $k(\theta)$ anisotropy and the consequences of our results on the relative weights of the s-wave and d-wave components in the superconducting order parameter of YBCO are discussed elsewhere[17].

In conclusion, we have shown that the angular variation of the thermal conductivity for a magnetic field applied parallel to the sample plane, was able to discriminate superconductors with similar geometrical shapes but with different microscopic gap structures.

\section{References}

1. D.A. Bonn et al.: Phys. Rev. Lett. 68, 2390 (1992); W.N. Hardy et al.: Phys. Rev. Lett. 70, 3999 (1993); Kuan Zhang et al.: Phys. Rev. Lett. 73, 2484 (1994)

2. Shen et al.: Phys. Rev. Lett. 70, 1553 (1993); H. Ding et al.: Phys. Rev. Lett. 74, 2784 (1995)

3. J.A. Martindale et al.: Phys. Rev. Lett. 68, 702 (1992); Bulut et al.: Phys. Rev. Lett. 68, 706 (1992)

4. D.A Wollman et al.: Phys. Rev. Lett. 71, 2134 (1993); Tsuei et al.: Phys. Rev. Lett. 73, 593 (1994)

5. James Annett, Nigel GoldenFeld, Anthony J. Leggett: [condmat/9601060], to appear in Physical Properties of High Temperature Superconductors, Vol. 5, D.M. Ginsberg (ed.), World Scientific, Singapore, 1996

6. B. Lussier, B. Ellman, L. Taillefer: Phys. Rev. Lett. 73, 3294 (1994); Phys. Rev. B53, 5145; A. Huxely et al.: Phys. Lett. A 209, 365 (1995)

7. S.D. Peacor et al.: Phys. Rev. B 44, 9508 (1991)

8. R.C. Yu et al.: Phys. Rev. Lett. 71, 1657 (1993)

9. K. Krishana, J.M. Harris, N.P. Ong: Phys. Rev. Lett. 75, 3529 (1995)

10. P.J. Hirschfeld, W.O. Putikka: Phys. Rev. Lett. 77, 3909 (1996)

11. M.B. Salamon et al.: J. Superconductivity 8, 449 (1995)

12. F. Yu et al.: Phys. Rev. Lett. 74, 5136 (1995)

13. R. A. Klemm et al.: Phys. Rev. Lett. 77, 3056 (1996)

14. Kazumi Maki: Phys. Rev. 158, 397 (1967)

15. J. Lowell, J.B. Sousa: J. Low Temp. Phys. 3, 65 (1970)

16. A.F. Andreev: Sov. Phys. JETP 19, 1228 (1964)

17. H. Aubin et al.: to be published 